\title{
Co-Infection with Hepatitis B Virus and Hepatitis C Virus
}

\author{
Heloísa Pedrosa Mitre and João Silva de Mendonça \\ Infectious Diseases Division of the Hospital for State Civil Servants; São Paulo, SP, Brazil
}

The hepatitis B virus (HBV) and the hepatitis C virus (HCV) share common transmission pathways. Therefore, co-infection can be expected. The World Health Organization estimates that, worldwide, 170 million people are infected with $\mathrm{HCV}$, and 350 million people are infected with HBV. However, the number of individuals co-infected with both viruses is unknown.

Although various studies have evaluated small numbers of co-infected individuals, the inclusion criteria, parameters assessed, and study designs are not uniform. In addition, ethnicity, local epidemiology, and viral genotypes are also diverse. Therefore, the conclusions of a specific study, in principle, should not be widespread.

In cases of co-infection with $\mathrm{HBV}$ and $\mathrm{HCV}$, the replication of either virus can be inhibited, just as either virus can be dominant or the dominance can alternate between the two. It is more common for HBV to appear to be suppressed by HCV. The chronologies of the two infections have an influence on which virus will be dominant. Molecular biology techniques (to determine levels of HCV RNA and HBV DNA) have facilitated the definition of the interaction between the two viruses.

Co-infections can appear in various manners:

a) Simultaneous acute infection with HBV and HCV: presupposes same source and transmission pathway. The number of studies is small, but indicates that the interaction between the two viruses is similar to that which occurs in chronic infections. There are descriptions of cases in which there is a delay in the identification of the Hepatitis B surface antigen (HBsAg), lower levels of alanine aminotransferase (ALT), and lower HBV antigenemia, which can be attributed to suppression of HBV activity by HCV.

b) Superinfection by one virus, the other virus being chronically present: it should be suspected above all in individuals with risk factors, such as the use of illicit intravenous drugs, multi-transfused individuals, and those living in areas of high HBV prevalence.

Superinfection by HCV: infection by HCV in a patient that is already infected with HBV. This is known in Asian countries, where the prevalence of HBV is high.

The viral suppression of the HCV may occur, although the viral suppression of the HBV is more frequent, noted by lower levels of HBV DNA and lower DNA polymerase activity, as well as by the hepatic expression of HBsAg and hepatitis B core antigen (HBcAg), clearance of the hepatitis B e antigen (HBeAg) or even of the HBsAg.

The HCV core antigen seems to affect the transcription of $\mathrm{HBV}$ and, as a result, its replication, which is reported to be more accentuated for the HCV genotype 1.

The Brazilian Journal of Infectious Diseases $\quad$ 2007;11 (5) Suppl. 1:33-35. (C) 2007 by The Brazilian Journal of Infectious Diseases and Contexto Publishing. All rights reserved.
After HBV clearance, HCV can persist, resulting in chronic hepatitis. In addition, there is the possibility of evolution to severe disease, with a risk of death.

Superinfection by HBV: infection by HBV in an individual that is already chronically infected with HCV. The HCV RNA levels are lower, and, in a study conducted in Italy, HCV clearance was higher in co-infected individuals (71\%) than in mono-infected individuals (14\%).

However, the HBV DNA levels can be lower than those of mono-infected individuals, indicating HCV interference. Therefore, one virus can induce the clearance of another.

The evolution to severe forms of the disease, with fulminant hepatitis profiles, has also been described.

c) Asymptomatic infection with HBV: there are reports of patients infected with HCV, with low levels of HBV DNA, reactive anti-HBc, however, non-reactive $\mathrm{HBsAg}$, HBeAg, anti-HBe, and anti-HBs, configuring co-infection with HCV with asymptomatic HBV.

These individuals evolve with high ALT levels and high histological activity. There are reports in which biopsies from such patients were evaluated, and cirrhosis was found in $33 \%$ of the cases, compared with $19 \%$ for patients presenting chronic mono-infections.

The data suggest that the evolution of the disease is more severe in co-infected individuals.

There are various potential outcomes:

a) Clearance of both viruses: negative HBsAg and, eventually, appearance of anti-HBs. The anti-HCV remains reactive. However, both HBV DNA and HCV RNA become undetectable. This is the best evolution for both viruses.

b) Fulminant hepatitis: several studies indicate that individuals co-infected with HBV and HCV have a higher risk of evolution to fulminant hepatitis. In a prospective study involving patients with acute hepatitis C, of those who died, 23\% were chronically infected with HBV, whereas only $2.9 \%$ were infected with HBV $(\mathrm{OR}=10.2)$.

In a study conducted in France, among 40 patients with fulminant or sub-fulminant hepatitis, $12.5 \%$ presented acute hepatitis B or C, and 7.5\% presented superinfection with HCV.

c) Chronic hepatitis: of all possible events, this is the one that occurs most frequently and presents treatment possibilities. Among the chronic cases of hepatitis, several immunological and molecular-biological profiles can be identified:

Active HBV and HCV: detectable HBV DNA and HCV RNA in serum. This situation has higher possibility of evolution to cirrhosis and hepatic decompensation. In these cases, treatment may be considered, either with interferon (IFN) + ribavirin (RBV) or IFN + lamivudine (LMV). 
Inactive $\mathrm{HBV}$ and active $\mathrm{HCV}$ : with evolution to undetectable HBV DNA and detectable HCV RNA. If treatment is indicated, $\mathrm{IFN}+\mathrm{RBV}$ is the treatment of choice.

Active HBV and inactive HCV or previous infection with $\mathrm{HCV}$ : with reactive $\mathrm{HBsAg}$ and $\mathrm{HBeAg}$, detectable HBV DNA and undetectable HCV RNA.

If indicated, therapeutic options may be IFN or IFN + LMV. Cirrhosis: co-infected individuals have a higher risk of evolution to cirrhosis if compared with mono-infected individuals (44\% and 21\%, respectively) as well as a higher risk of chronic hepatitis decompensation.

Hepatocellular carcinoma: evidence indicates that the possibility of evolution to hepatocellular carcinoma (HCC) is higher in co-infected individuals. A prospective study comprising 290 individuals with cirrhosis concluded that, in the univariate and multivariate analyses, co-infection with $\mathrm{HBV}$ and $\mathrm{HCV}$ is a predictive factor for the development of HCC. These conclusions were confirmed in subsequent studies, in which the incidence of HCC in individuals coinfected with HBV and HCV was 2 and 3.7 cases/100 individuals/year, respectively.

The cumulative risk of HCC development in 10 years was $45 \%$ in co-infected individuals, $16 \%$ in individuals monoinfected with $\mathrm{HBV}$, and $28 \%$ in individuals mono-infected with HCV.

\section{Treatment}

The treatment guidelines have been defined in monoinfected individuals, among others, by professional associations of liver researchers, such as the Asian-Pacific Association for the Study of the Liver, the European Association for the Study of the Liver, and the American Association for the Study of Liver Diseases. According to these guidelines, the choice of pegylated interferon over conventional interferon was defined in association with ribavirin in cases of chronic infection with $\mathrm{HCV}$, and, in isolation, in cases of chronic infection with HBV, when the administration of antiviral drugs is also considered.

On the other hand, the treatment of individuals co-infected with $\mathrm{HBV}$ and $\mathrm{HCV}$ is complex due to the interaction between both viruses, and between both viruses and the host immunologic system. Standards for these treatments are not yet available. However, the therapeutic regimen should be carefully chosen and should be based on serologic markers, viremia levels, histology, and, above all, definition of the dominant virus.

Individuals with uncompensated cirrhosis should be referred to facilities specializing in liver transplantation.

\section{Interferon}

Since IFN is an immunomodulating drug, with antiviral and antiproliferative effect, it is effective against HBV and HCV. It is also the most widely studied therapeutic option. The first study in co-infected individuals, carried out in the 1990s, suggests that higher doses (9 MU 3 times a week) are more effective for the clearance of $\mathrm{HBV}$ or HCV.
If suppression of one of the viruses is achieved, there is the possibility of reactivation of the other, since the suppressor effect of the former has been removed. Pegylated IFN, which is more effective in mono-infected individuals, can have a similar effect in co-infected individuals.

In the infection with $\mathrm{HCV}$ and asymptomatic HBV infection, the histological changes and activity are typically pronounced. The response to treatment is less favorable and respondents present recurrence (downregulation?).

IFN + RBV: this is the regimen that performs best in individuals mono-infected with HCV. There have been various studies, involving small samples of co-infected individuals, reporting rates of sustained biochemical response and sustained virologic response (SVR) that are comparable to those of mono-infected individuals, especially if HCV is the dominant virus.

Due to the viral interaction, it is extremely important that HBV activity is monitored. It is known that at least half of the patients present $\mathrm{HBV}$ reactivation at the beginning of the treatment, and that $45 \%$ will have 'flare-ups'. Therefore, we should pay attention to HBV, even if HBV DNA is undetectable at the beginning of the treatment. Over the course of the disease, HBV DNA can become undetectable in 10\% to $30 \%$ of the patients.

IFN + LMV: small studies suggest that the addition of LMV is useful, above all in patients with active HBV. One such study described eight HBeAg-reactive patients, in whom HBV DNA and HCV RNA were detected, submitted to a 12-month course of IFN + LMV, followed by an additional six months of treatment with LMV. In approximately one-third of the patients, there was HBV and HBeAg clearance. In half of the patients, there was normalization of ALT, HCV clearance, and an SVR.

It would be premature to venture an opinion on what is the most appropriate regimen, considering that further studies, involving larger patient samples, are still needed.

Adefovir and entecavir: there have been no studies involving co-infected individuals. These might be good options in patients with dominant HBV.

Liver transplantation: little experience with co-infected individuals.

There are reports of higher survival compared with individuals mono-infected with HBV, with suggestion of the benefit of HCV suppression over HBV in immunosuppression after transplantation.

In order to define the risks and benefits, it is necessary to await the results of further, larger studies.

\section{Triple Co-Infection}

a) HBV, HCV, and hepatitis D virus (HDV): only patients who are already infected with HBV can acquire HDV. The triple infection can occur in certain geographic areas where the prevalence of infection with HBV is high.

The evolution to severe disease is described, the desired treatment being that that consists of high doses of IFN for a long period and, still, with poor responses. 
There are few relevant studies in the literature, and all of those involved small study samples, which precludes the standardization of the procedure in these cases. There are reports of treatment of seven patients with achievement of sustained biochemical response in two patients, and worsening with side effects in two other patients.

b) HBV, HCV, and HIV: in these cases, the complexity of the $\mathrm{HBV} / \mathrm{HCV}$ interaction is added to the effect of the HIV on the host immunologic system.

It is known that, in HCV/HIV and HBV/HIV co-infections, the evolution of the hepatic disease is faster and potentially more severe. In addition, after the introduction of the highdose anti-HIV therapy known as highly active antiretroviral therapy, the mortality rate for chronic liver diseases has increased.

There are few studies on the treatment of individuals coinfected with HBV, HCV, and HIV. The rate at which an SVR is achieved has been reported to be $17 \%$ with the use of IFN, compared with $25 \%$ for IFN + RBV.

It must be borne in mind that, due to the frequent use of LMV in the antiretroviral therapy for HIV, HBV resistance to LMV is high in co-infected individuals.

In one small study, the effect against the dominant virus, $\mathrm{HBV}$ or HCV, did not lead to the reactivation of the other, except in one case involving a patient with a very low CD4 lymphocyte count.
Studies of new antiviral drugs that are more effective against HBV (tenofovir, entecavir, adefovir, etc.) in HBV/ $\mathrm{HIV}$ or HBV/HCV/HIV co-infected individuals might inform therapeutic decisions regarding the complex treatment of co-infections involving HBV.

\section{References}

1. Chakravarti A., et al. Characteristics of dual infection of hepatitis B and $\mathrm{C}$ viruses among patients with chronic liver disease: a study from tertiary care hospital. Trop Gastroenterol 2005;26:183-7.

2. Crockett S.D., et al. Natural story and treatment of hepatitis B virus and hepatitis $\mathrm{C}$ virus coinfection. Ann Clin Microbiol Antimicrob 2005;4:1-12.

3. French A.L., et al. Isolated hepatitis B core antibody is associated with HIV and ongoind but not resolved hepatitis $\mathrm{C}$ virus infection in a cohort of US women. JID 2007;195:1437-41.

4. Liu Z., et al. Hepatitis B virus (HBV) and hepatitis C virus (HCV) dual infection. Int J Med Sci 2006;3:57-62.

5. Sagnelli E., et al. Virologic and clinical expressions of reciprocal inhibitory effect of hepatitis B, C, and Delta viruses in patients with chronic hepatitis. Hepatology 2000;32:1106-10.

6. Sagnelli E., et al. HBV superinfection in hepatitis C cirus chronic carriers, viral interaction, and clinical course. Hepatology 2002;36:1285-91.

7. Sagnelli E., et al. Hepatitis C virus superinfection in hepatitis B virus chronic carriers: a reciprocal viral interaction and a variable clinical course. J Clin Virol 2006;35:317-20.

8. Soriano V., et al. Treatment of chronic hepatitis B or C in HIVinfected patients with dual viral hepatitis. JID 2007;195:1181-3.

9. Wang Y.M., et al. Suppression of hepatitis C virus by hepatitis B virus in coinfected patients at the National University Hospital of Singapore. J Gastroenterol 1999;34:481-5. 